\title{
REAL-TIME MOUTH DEFECTS DETECTION ON MILITARY CARTRIDGE CASES
}

\author{
Semra Aydin ${ }^{1}$, Refik Samet $^{2}$ and Omer Faruk Bay ${ }^{3}$ \\ ${ }^{1}$ Education of Computer and Electronics, Gazi University, Ankara, Turkey \\ ${ }^{2}$ Department of Computer Engineering, Ankara University, Ankara, Turkey \\ ${ }^{3}$ Department of Electric and Electronics Engineering, \\ Gazi University, Ankara, Turkey
}

\begin{abstract}
A military cartridge includes four elements; case, capsule, ammunition and powder. While manufacturing, defects may occur in the case. These defects should be detected and the defected cases should be separated. Defects could occur in the mouth, surface and primer parts of the case. This paper proposes the methodology that involves the real-time inspection of the defects in the mouth part of the cases using image processing techniques. The algorithms of the proposed methodology were implemented on real images and the obtained results have showed that common defects such as split and dent defects occurring on the mouth part of the case can be detected with high accuracy.
\end{abstract}

\section{KEYWORDS}

Military cartridge, case mouth defects, defect detection, circle detection.

\section{INTRODUCTION}

Computer vision systems are widely used in industrial applications to inspect defects in real-time. These systems control the real-time production lines by processing the images taken by cameras and separate defected products. Due to computer vision systems, the production capacity and quality of products increase and the product cost and inspection time decrease.

Main functions of the computer vision systems are fulfilled by image processing techniques. Some industrial products with cylindrical and metal surfaces need to be inspected in moving state. Inspection of dynamic and complex products using real-time image processing techniques is a nontrivial problem.

Image processing to inspect the products with cylindrical and metal surfaces has some challenges, which are related to quality of images taken in moving state. The first challenge is related to the glitters problem during illumination. In order to get images with high quality, a special ring lightning should be used. Due to cylindrical and metal surfaces, some part of the products can be glittered. The second challenge is related to the moving state of products. It is hard to capture images of moving product in the same position. The third challenge is the cylindrical surfaces itself. In order to inspect the whole 360-degree of the surface, it is necessary to capture the product surface on the fly. The pixel intensity is changed depending on the distance between 
camera and cylindrical surface of the product. All of these challenges make image processing more complex.

The military cartridge consists of four parts: case, capsule, ammunition and powder. The military cartridge case consists of three elements: mouth, cylindrical surface and primer (Fig. 1).

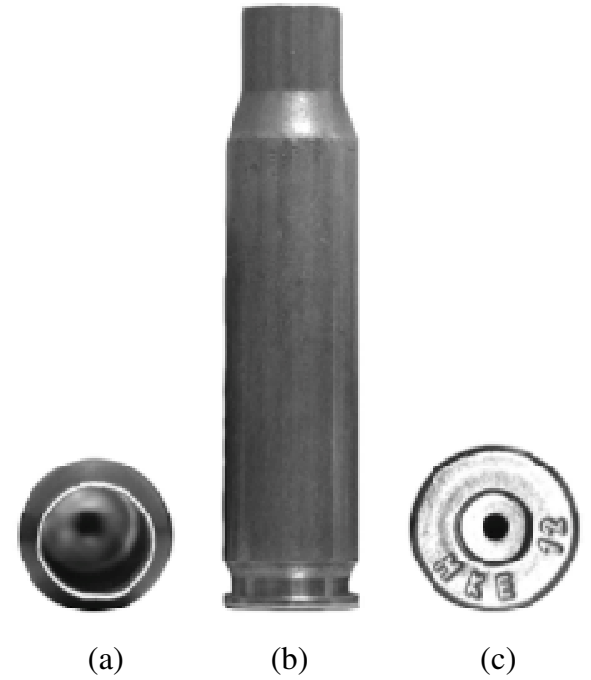

Figure 1. Parts of military cartridge case: (a) mouth, (b) cylindrical surface, (c) primer.

This study investigates the defects on the mouth of the cases where different defects, like split and dent defects, could occur. These defects are defined by NATO standards [1,2]. At the final stage of the production process, defects should be inspected and then the defected ones should be separated. These operations should be done in real-time.

In this study, a computer vision system for the inspection of moving cartridge case is used. In this system, one camera is used for each of the mouth and the primer, and eight cameras are used for the cylindrical surface to take images. The images taken by the cameras are processed by realtime image processing algorithms to detect defects. The defected cases detected are separated at the final stage of computer vision processing.

This study proposes the methodology for real-time inspection of defects on the mouth part of the cases by image processing techniques.

The manuscript is organized as follows. In Section 2, related works are summarized. The detection methodology of defects is proposed in Section 3. Implementation of proposed methodology is described in Section 4. Finally, Section 5 concludes obtained results.

\section{RELATED WORKS}

In computer visual inspection systems, the defect detection algorithms are used to determine the defected products. Existing works use different algorithms to detect the defects on the different materials. The most common topic is related to textile materials. Kumar et al. [3] investigate various approaches for automated inspection of textured materials using Gabor wavelet features. Kumar [4] investigates different techniques for the detection of automated fabric defects. Chan and Pang [5] propose a method using Fourier transform to detect fabric defects. 
There are many studies related to the detection of metallic surface defects in automated inspection systems. Choi et al. [6] present a real-time defect detection algorithm for high-speed steel bar in coil. Frayman et al. [7] present a camera based machine vision system for the automatic inspection of surface defects in aluminium die casting using genetic algorithm. Karimi and Asemani [8] compare and evaluate different defect detection algorithms using various performance parameters. In the literature, various image processing techniques were used to detect defects on different kinds of materials. This paper adopts and uses existing techniques to detect defects on cylindrical and metal objects in real-time.

Following studies are related to circle detection which can be used to detect the defects in the circularity of objects. To detect the circles with image processing techniques, different algorithms can be used. In [9], an algorithm for edge and segment detection was proposed. This algorithm can detect the circles in real-time images. In [10], a circle detection algorithm for automatic sign detection and recognition was proposed. The authors in [11] have proposed another algorithm to detect circles on images. In [12], a review study related to Hough algorithm was reported. An efficient Hough transform for circle detection was proposed in [13]. It was reported that, Hough is a popular algorithm to detect the circles. But to the best of our knowledge, there are no studies related to implementation of Hough algorithm to the moving cylindrical and metal objects. This paper uses Hough algorithm for checking the circularity to detect the defects in circles of realtime images of cylindrical and metal objects captured in a moving state.

\section{MOUTH DEFECTS DETECTION METHODOLOGY}

Different defects such as split, dent, etc. can occur in the mouth part of military cases (Fig. 2).



(a)

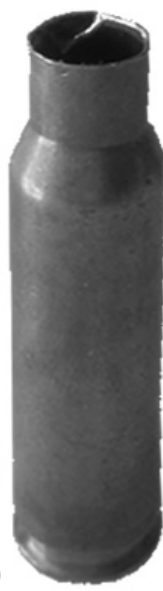

(b)

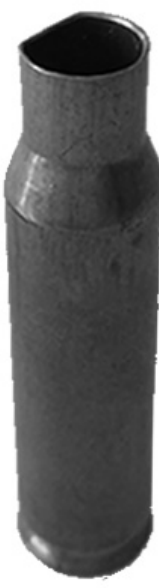

(c)

Figure 2. (a) Normal mouth, (b) mouth with split defect, (c) mouth with dent defect

The proposed methodology to detect the defects in the mouth part of the cases consists of the following stages:

- Preprocessing;

- Mouth processing;

- Split defect detection;

- Dent defect detection.

Block diagram of the entire defects detection methodology is shown in Fig. 3. 




Figure 3. The block diagram of proposed defect detection methodology

\subsection{Preprocessing}

Preprocessing stage includes four steps: 1) cropping; 2) noise reduction; 3) thresholding and 4) morphological operations (closing). 


\subsubsection{Cropping}

In real-time applications, the image processing performance should be optimized. For this, the size of captured raw images should be decreased. In other words, unnecessary part of raw images should be cropped.

\subsubsection{Noise Reduction}

Noise reduction is a process of removing noise from an image. Generally; every image has some noise that needs to be eliminated. Several techniques can be used to remove the noise from the image. Gaussian filter is widely used to reduce image noise.

\subsubsection{Thresholding}

Thresholding is a key step in defect detection process. The success of subsequence stages of methodology depends on the result of thresholding step [13, 14]. Mouth part of the captured image is glittered due to illumination. In order to get the glittered mouth in cropped image, bilevel thresholding should be used. At the result of bi-level thresholding, the glittered part of the mouth is denoted by white pixels and inside the mouth is filled by black pixels.

\subsubsection{Morphological Operations}

In order to improve the success, some morphological operations should be applied to the images. After thresholding, small discontinuities may appear. Closing operation can help eliminating such discontinuities.

\subsection{Mouth Processing}

Mouth processing stage includes three steps: 1) detection of the circle; 2) detection of the inner edge of the mouth and 3) drawing an edge of mouth.

\subsubsection{Cycle Detection}

Different algorithms can be used to detect the circle. Hough transform is a common algorithm used to detect geometry shape of the objects in image processing [15, 16]. At the results of Hough circle detection process, the center and the radius of the circle are defined. Center of the circle is denoted as $c(x, y)$ that is the pixel in the image. Radius is denoted as $r$.

\subsubsection{Detection of Inner Edge of Mouth}

First, the image obtained at thresholding step is scanned 360 degrees beginning from the center pixel of $c(x, y)$ toward to the inner edge of the mouth (Fig.4).

During scanning, 360 points detected on the inner edge of the mouth will be denoted as $C\left(X_{\varphi}, Y_{\varphi}\right)$. Here, $X_{\varphi}$ and $Y_{\varphi}$ are the coordinates of $C\left(X_{\varphi}, Y_{\varphi}\right)$ and calculated as follows:

$$
\begin{aligned}
& X_{\varphi}=x+\left(i_{\varphi} * \cos (\varphi * p i \div 180)\right) \\
& Y_{\varphi}=y+\left(i_{\varphi} * \sin (\varphi * p i \div 180)\right)
\end{aligned}
$$




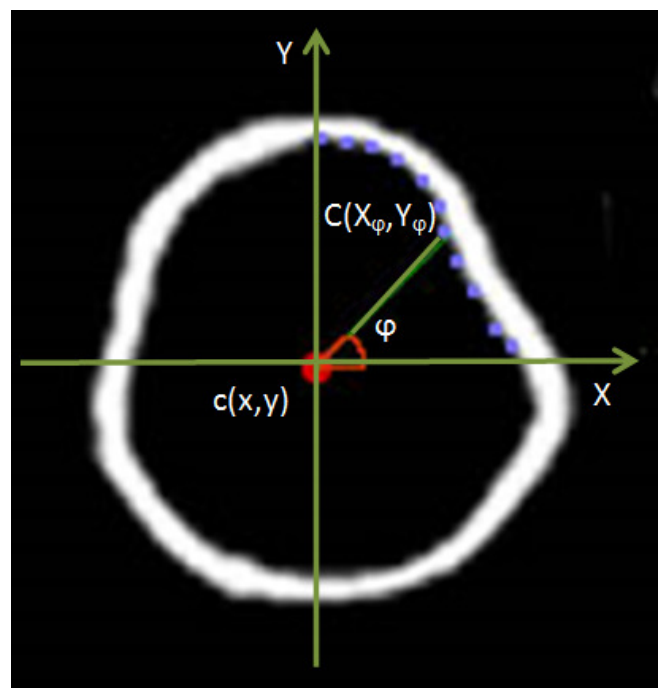

Figure 4. Detection of inner edge of mouth

where, $\varphi(\varphi=1,2, \ldots, 360)$ is the degree of the 360 points of $C\left(X_{\varphi}, Y_{\varphi}\right)$ on the inner edge of the mouth. Next, the distance $i_{\varphi}\left(i_{\varphi}=1,2, \ldots, I\right)$ between $c(x, y)$ and $C\left(X_{\varphi}, Y_{\varphi}\right)$ is calculated. $i_{\varphi}$ is defined as the minimum distance between $c(x, y)$ and $C\left(X_{\varphi}, Y_{\varphi}\right)$. Finally, $C\left(X_{\varphi}, Y_{\varphi}\right)$ and $i_{\varphi}$ are stored as an array and used for the next step.

\subsubsection{Drawing an Edge of Mouth}

By using the array obtained in the previous step, the edge of the mouth is drawn by marking the black pixels on the white background.

\subsection{Split Defect Detection}

Euclidean distance is used to measure the distance between $C\left(X_{\varphi}, Y_{\varphi}\right)$ and $C\left(X_{\varphi}+1, Y_{\varphi}+1\right)$. If the Euclidean distance is greater than $\Delta$ then the split defect on the mouth is present. $\Delta$ is the Euclidean distance for mouth of non-defected cases. If the Euclidean distance is less than $\Delta$ then it is necessary to go to the next stage of the methodology to detect the dent defect.

\subsection{Dent Defect Detection}

Dent defect detection stage includes three steps: 1) circle detection in the inner edge of the mouth; 2) calculation of the cumulative variance and 3) classification of the dent defect.

\subsubsection{Circle Detection on Inner Edge of Mouth}

Hough circle detection is applied to the inner edge of the mouth drawn in Section 3.2.3. The circle detected here is overlapped better with the mouth of the case with respect to the circle detected in Section 3.2.1. If Hough transform cannot detect any circle in the inner edge of the mouth, the circle detected in Section 3.2.1 is used.

\subsubsection{Calculation of Cumulative Variance}

The variance used for the detection and classification of the circular objects. Two different techniques are used to calculate the cumulative variance. 
a) In the first one, central point $c(x, y)$ and radius $r$ of the circle, detected in Section 3.2.1 by Hough algorithm, are used to calculate the cumulative variance. In order to calculate the cumulative variance of each degree of $\varphi(\varphi=1,2, \ldots, 360)$, the Euclidean distance between the same points on the circle detected in Section 3.4.1 and the edge of the mouth drawn in Section 3.2.3 is calculated. The sum of all the square of Euclidean distance values for 360 degrees gives us the cumulative variance.

b) In the second technique, the average distance between the points on the edge of the mouth drawn in 3.2.3 and central point $c(x, y)$ is calculated. Then, the Euclidean distance between the points on the circle and the points on the edge of the mouth drawn in 3.2.3 is calculated. Finally, the sum of square of all 360 Euclidean distance values for 360 degrees gives us the cumulative variance.

\subsubsection{Classification of Dent Defects}

When the mouth of the cases is fully circular, these kind of cases are classified as non-defected. If the calculated cumulative variance is less than $\delta$ then the cases are also classified as nondefected. On the other hand, if cumulative variance is greater than $\delta$, the cases are classified as defected.

\section{IMPLEMENTATION}

All experiments were performed on platform with i7 $2.4 \mathrm{GHz}$ CPU, 8 GB RAM and Windows 7. All algorithms used in this study were coded in Visual Studio C++. All images were captured by PointGrey Flea 3 GigE 2.0 MP and Wordop $180 \mathrm{~mm}$ ring red colored led light was used to illuminate the cases.

According to the real-time conditions, five cases per second should be handled. So, each case should be processed during 200 milliseconds. Proposed algorithms should meet the real-time conditions.

A case inspection system was developed to capture images and to separate defected cases. An example of image captured on the conveyor band is shown in Fig. 5.

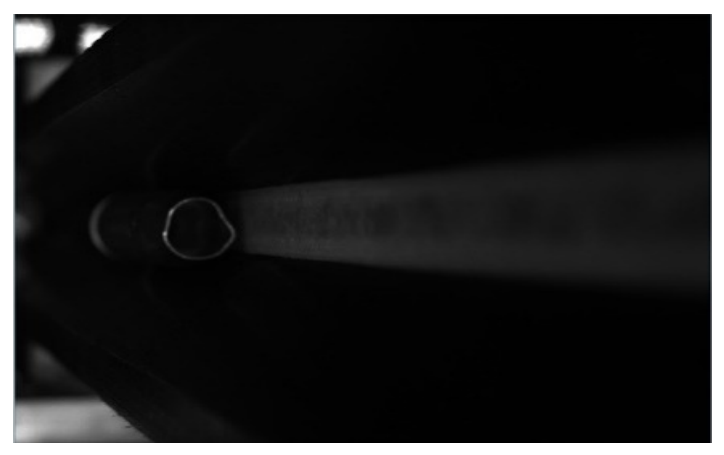

Figure 5. Original image

The implementation results of the proposed methodology on the real images are described below.

\subsection{Preprocessing}

The original image resolution is $1600 \times 1200$ pixels. After cropping, the image with resolution 350x350 pixels was obtained (Fig. 6). 




(a)

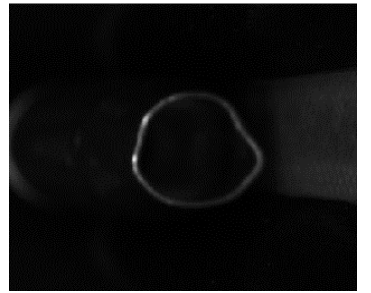

(b)

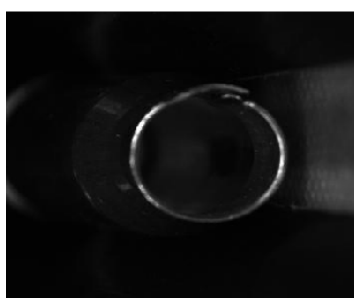

(c)

Figure 6. Cropped mouth of non-defected case (a); dent defected case (b); and split defected case (c)

Gaussian filter was applied to the cropped images for noise reduction (Fig. 6).

Binary thresholding was done based on the Otsu threshold value. Following the thresholding, closing process was carried out and small discontinuities in the mouth of the case were closed (Fig. 7).



(a)

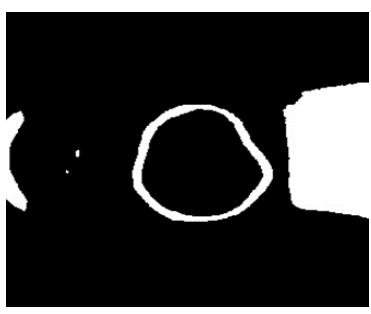

(b)

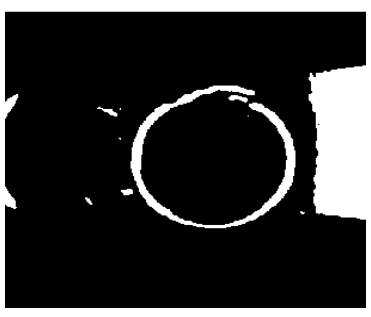

(c)

Figure 7. The results after thresholding and closing operation: non-defected mouth (a); dent defected mouth (b); and split defected mouth (c)

\subsection{Mouth Processing}

\subsubsection{Circle Detection}

Hough circle detection algorithm was used to detect the circle in the mouth of the case. The center and the radius of circle were found using this algorithm. The red circle was obtained in Fig. 8.

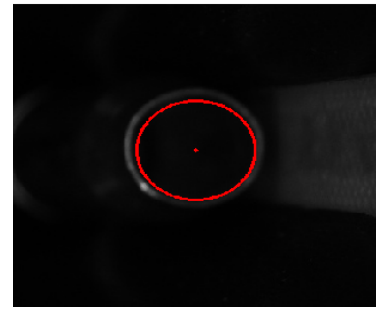

(a)

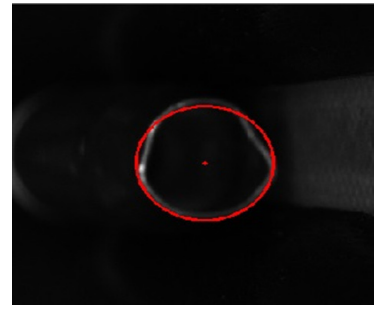

(b)



(c)

Figure 8. Detected circles of non-defected mouth (a); dent defected mouth (b); and split defected mouth (c)

\subsubsection{Detection of Inner Edge of Mouth:}

Inner edge of the mouth was found and illustrated with blue color in Fig. 9. 


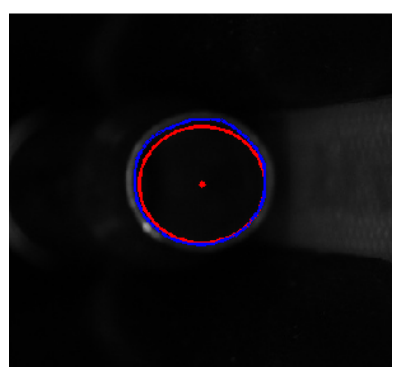

(a)

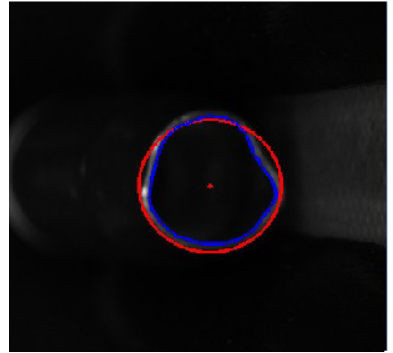

(b)

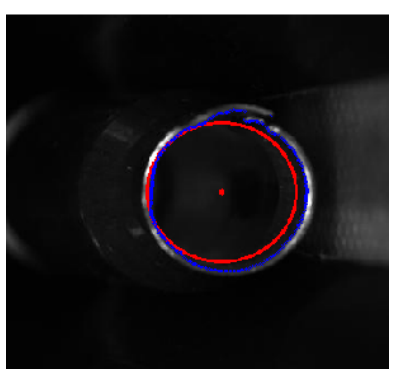

(c)

Figure 9. Blue colored inner edge of non-defected mouth (a); dent defected mouth (b); and split defected mouth

\subsubsection{Drawing the inner edge of mouth}

The inner edge of mouth was reconstructed by marking the pixels of obtained array in Section 3.2.2 as black on the white background (Fig. 10).

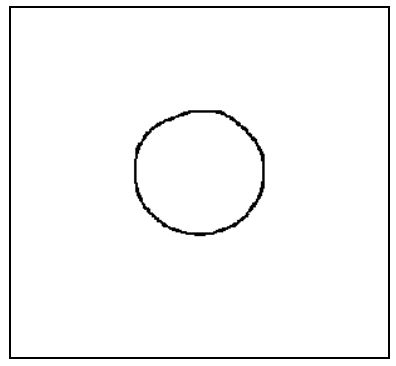

(a)

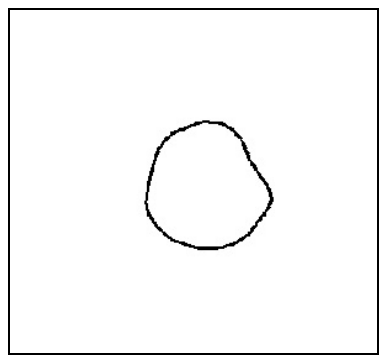

(b)



(c)

Figure 10. Reconstructed non-defected mouth (a); dent defected mouth (b); and split defected mouth (c)

\subsection{Split Defect Detection}

As seen from Fig. 9 (a) and (b), there is no interruption on the edge of the mouth. Suppose that $\Delta=2$. The distance between neighbor pixels of mouth edge is calculated as 1-2. This means that the case is non-defected. On the other hand, there is an interruption on the edge of the mouth in Fig. 10 (c). For this case, the some distance was bigger than the value $\Delta=2$ for split defected mouth. This means that the case is defected.

\subsection{Dent Defect Detection}

\subsubsection{Hough circle detection on the inner edge of the mouth:}

Fig. 11 shows the first (red) (Section 3.2.1) and second (yellow) (Section 3.4.1) circles obtained by Hough circle detection. The blue line is the inner edge of the mouth. During the second circle detection, the center of the mouth of the case was found more accurately.

\subsubsection{Calculation of cumulative variance}

The yellow line is perfect circle and the blue line is the mouth of the case. Cumulative variance was calculated between the yellow and the blue lines. 




(a)

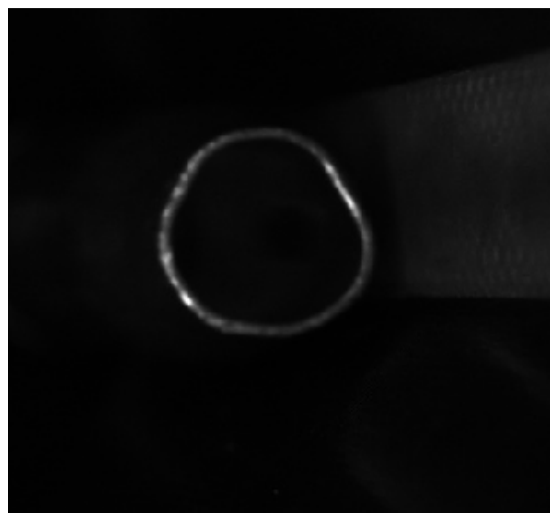

(c)



(b)



(d)

Figure 11: Non-defected mouth (a); circles detected on non-defected mouth (b); dent defected mouth (c); circles detected on dent defected mouth (d)

\subsubsection{Classification of dent defect}

If the cumulative variance is bigger than determined value of the mouth, the case will be defined as defected. Otherwise the case will be defined as non-defected.

\section{RESULTS}

The results obtained were evaluated using confusion matrix theory [17, 18] which has four categories. True Positives (TP) mean the defected cases are detected as defected. False Positives (FP) mean the non-defected cases are detected as defected. True Negatives (TN) mean the nondefected cases are detected as non-defected. False Negatives (FN) mean the defected cases are detected as non-defected. The confusion matrix is shown in Table 1.

Table 1. Confusion matrix

\begin{tabular}{|l|l|c|c|}
\hline \multirow{4}{*}{$\begin{array}{c}\text { Actual } \\
\text { military case }\end{array}$} & Desult of algorithm \\
\cline { 2 - 4 } & Defected & TP & Non-defected \\
\cline { 2 - 4 } & Non-defected & FP & TN \\
\hline
\end{tabular}


The results are evaluated by error rate, recall, precision and F-measurement.

Error rate is defined as follows:

$$
E r r=\frac{F N+F P}{T P+F N+F P+T N}
$$

Recall is defined as follows:

$$
R e C=\frac{T P}{T P+F N}
$$

Precision is defined as follows:

$$
\text { Pre }=\frac{T P}{T P+F P}
$$

F-measurement is defined as follows:

$$
F=\frac{2 \times \text { Rec } \times \text { Pre }}{\text { Rec }+ \text { Pre }}
$$

$50 \%$ non-defected and $50 \%$ defected images were used during experiments.

Table II shows the results obtained using the cumulative variance calculated on the base of the radius of the circle by Hough algorithm (Section 3.4.2 (a)).

Table 2. Results on the base of the radius value

\begin{tabular}{|l|l|c|c|}
\hline \multirow{2}{*}{$\begin{array}{l}\text { Actual } \\
\text { defects of } \\
\text { case }\end{array}$} & Defected & Defected & Non-defected \\
\cline { 2 - 4 } & Non-defected & $48 \%$ & $2 \%$ \\
\cline { 2 - 4 } & Nosult of algorithm \\
\hline
\end{tabular}

Table III shows the results obtained using the cumulative variance calculated on the base of the average distance (Section 3.4.2 (b)).

Table 3. Results on the base of the average distance

\begin{tabular}{|c|l|c|c|}
\hline & \multicolumn{3}{|c|}{ Result of algorithm } \\
\hline \multirow{2}{*}{$\begin{array}{c}\text { Actual } \\
\text { defects of } \\
\text { case }\end{array}$} & Defected & $48 \%$ & Defected \\
\cline { 2 - 4 } & Non-defected & $8 \%$ & $2 \%$ \\
\cline { 2 - 4 } & Nocted \\
\hline
\end{tabular}


Table IV shows the results of error rate, recall, precision, and F-measurement calculated by using Tables II and II and Eq. (3)-(6).

Table 4. Results of proposed methodology

\begin{tabular}{|c|c|c|c|c|}
\hline & Error Rate & Recall & Precision & F-measurement \\
\hline Radius & $12 \%$ & 96 & 82 & 88 \\
\hline Average & $10 \%$ & 96 & 85 & 90 \\
\hline
\end{tabular}

As seen from Table IV, according to the values of metrics, the split and dent defects occurring on the mouth part of the case were detected with high accuracy in comparison with existing manual inspection system where the rates of the above listed metrics are very low. As seen from Tables II and III, the values of FN are $2 \%$ which means that very small number of defected cases is detected as non-defected. The other finding is related to FP which is calculated as $10 \%$ and $8 \%$. This means that a small number of non-defected cases was defined as defected. By improving the quality of online images the values of these metrics can be increased.

\section{CONCLuSiON}

This paper has proposed a methodology to detect defects in the mouth part of military cartridge cases. At all stages of the proposed methodology, special algorithms taking into account the challenges listed in introduction section were proposed.

The proposed methodology was implemented using real images. The results obtained showed that $96 \%$ of the defected cases were detected and separated in real-time. Experiment results have showed that the proposed algorithm guarantees the condition of the real-time processing and accuracy of detection.

In the future work, parallel programming techniques will be used to increase the accuracy rate and to decrease processing time.

\section{ACKNOWLEDGEMENTS}

This work has been funded by Ministry of Science, Industry and Technology of Turkey under grant San-Tez 0018.STZ.2013-1.

\section{REFERENCES}

[1] MIL-STD-651, Military Standard-Visual Inspection Standards for 20mm Ammunition and Components, Department of Defense, USA, 18 October 1962.

[2] MIL-STD-636, Military Standard-Visual Inspection Standards for Small Arms Ammunition Through Caliber .50, Department of Defense, USA, 5 June 1958.

[3] A. Kumar, and G.K.H. Pang, (2002) "Defect Detection in Textured Materials Using Gabor Filters", IEEE Transactions On Industry Applications, Vol. 38, No. 2, pp425-440.

[4] A. Kumar, (2008) "Computer-Vision-Based Fabric Defect Detection: A Survey", IEEE Transactions On Industrial Electronics, Vol. 55, No. 1, pp348-363.

[5] C. Chan, and G.K.H. Pang, (2000) "Fabric Defect Detection by Fourier Analysis", IEEE Transactions On Industry Applications, Vol. 36, No. 5, pp1267-1276. 
[6] S. H. Choi, J.P.Yun, B Seo, Y. S Park, and S. W. Kim, (2007) "Real-Time Defects Detection Algorithm for High-Speed Steel Bar in Coil", World Academy of Science, Engineering and Technology, Vol. 25, pp66-70.

[7] Y.Frayman, H. Zheng, and S. Nahavandi, (2006) "Machine Vision System for Automatic Inspection of Surface Defects in Aluminum Die Casting", Journal of Advanced Computational Intelligence and Intelligent Informatics, Vol. 10, No. 3, pp281-290.

[8] M. H. Karimi, and D. Asemani, "Surface defect detection in tiling Industries using digital image processing methods: Analysis and evaluation", ISA Transactions, in press

[9] C. Topal, and C. Akınlar, (2012) "Edge Drawing: A combined real-time edge and segment detector", J. Vis. Commun. Image R., Vol. 23, pp862-872.

[10] H. Gündüz, S. Kaplan, S. Günal, and C. Akınlar, (2013) "Circular traffic sign recognition empowered by circle detection algorithm", 978-1-4673-5563-6/13.

[11] B. Yuan, and M. Liu, "Power histogram for circle detection on images", Pattern Recognition, in press.

[12] P. Mukhopadhyay, and B. B. Chaudhuri, (2015) "A survey of Hough Transform", Pattern Recognition, Vol. 48, pp 993-1010.

[13] R. Samet, O.F. Bay, S. Aydin, S. Tural, A. Bayram, (2015) "Performance Analysis of GPU-based Parallel Image Segmentation using CUDA", ICAT'15 (International Conference on Advanced Technology \& Sciences), Antalya, Turkey.

[14] R. Samet, O.F. Bay, S.Aydın, (2015) "Real-Time Image Processing Applications on Multicore CPUs and GPGPU”, The 2015 International Conference on Parallel \& Distributed Processing Techniques \& Applications, Las Vegas USA.

[15] L. Jiang, (2012) "Efficient randomized Hough transform for circle detection using novel probability sampling and feature points", Optik, Vol. 123, pp1834-1840.

[16] R. Samet, A. Bayram, S. Tural, S. Aydin, (2016) "Primer Defects Detection on Military Cartridge Cases", NICOGRAPH International 2016. The 15th annual international conference, Hangzhou, China.

[17] J.R. Parker, (2001) "Rank and response combination from confusion matrix data", Information Fusion, Vol. 1, No. 2, pp113-120.

[18] G. Batista, R. C. Prati, M. C. Monard, (2004) "A study of the behavior of several methods for balancing machine learning training data”, ACM SIGKDD Explorations Newsletter, Vol. 6, No. 1, pp20-29.

\section{AUTHORS}

Semra Aydin received her B.S. degree in Computer Systems Education from Gazi University, Turkey. She received the M.S. degree in Graduate School of Natural and Applied Sciences from Gazi University about distributed computing. She has been studying on the Ph.D. in Computer Science since 2010, at Gazi University. Her research interests focus on paralel computing, image processing, multicore processing and GPU programming.

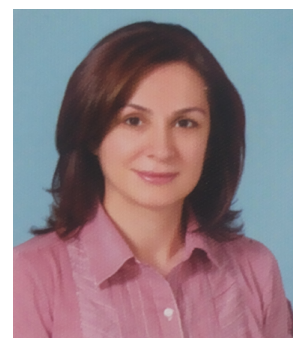


Refik Samet received the BS and MS degrees in automation and control in technical systems from the National Technical University of Ukraine "Kyiv Polytechnic Institute" in 1983 and the $\mathrm{PhD}$ degree in computer science from the Institute of Control Sciences of Russian Academy of Sciences in 1992. He is professor in the Department of Computer Engineering, Ankara University. His current research interests include fault-tolerant systems analysis, parallel systems, image/video processing, mobile applications. He has been a member of the IEEE and the Computer Society since 2000.

Omer Faruk Bay received the B.Sc. degree in Electrical and Electronics Education from Gazi University, Turkey in 1985, M.Sc. and Ph.D. degrees in Electronics Engineering from Erciyes University, Turkey in 1992 and 1996 respectively. He is a full professor at the Department of Electronics and Computer Education in Gazi University. His research interests include artificial intelligence and their applications; control and instrumentation; intelligent systems, BCI for home automation and image processing. He has been worked at some national and international Projects as a manager and researcher. He has published more than 85 articles and published one book.
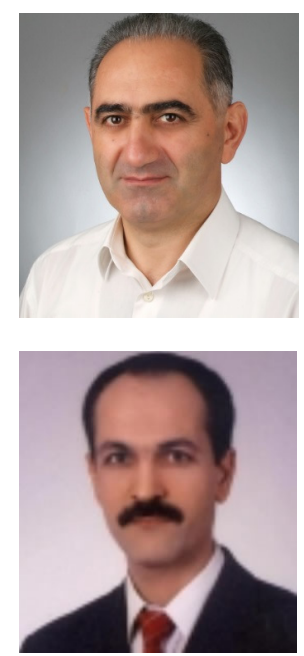